\title{
Dysfunctional Voiding: Does a validated urine color scale correlate with dysfunctional voidin severity score?
}

Katharina Mitchell, MD', Chad Crigger, MD, MPH'', Tyler Overholt, MD', John Barnard, MD'1, Tyler Trump, MD¹, Hilary Morley, MD², Osama Al-Omar, MD'1

ABSTRACT

Dysfunctional voiding (DV) affects approximately $40 \%$ of patients populating pediatric urology clinics. We administered the Dysfunctional Voiding Symptom Score [DVSS] survey and dehydration severity chart (Urine Color Chart [UCC]) to toilet trained pediatric patients and compared DV patients to patients presenting with non-urologic concerns. We compiled 29 DV patients (ages 3-15, 20 females, 9 males, 29 Caucasian) and 21 non-urologic patients (ages 2-17, 11 males, 10 females, 1 Hispanic, 1 African American, 20 Caucasian) from 2016 to 2018 . The DV population had significantly higher scores in $7 / 10$ items within the DVSS and overall scores compared to non-urologic patients $p<$ $0.05, p=0.0004$ respectively. This suggests the DVSS results yield significantly different results among DV and non-DV patients.

\section{KEYWORDS}

Dysfunctional Voiding, Pediatric Urology, Urine Color
Author affiliations are listed at the end of this article.

Correspondence to: Katharina Mitchell, MD West Virginia University Department of Urology kmm0051@hsc.wvu.edu

\section{INTRODUCTION}

Dysfunctional voiding (DV), defined as abnormal coordination between the detrusor muscle and the urinary sphincter and/or pelvic floor in the absence of neurologic disease, is a common pediatric diagnosis thought to affect approximately $40 \%$ of the patients that populate pediatric urology clinics. ${ }^{1}$ While the etiology is not entirely understood, DV has historically been considered a product of maladaptive, habitual behaviors. However, there is some debate on this theory due to a lack of evidence in support of a "learned" label, as well as concrete proof beyond any conceivable doubt that there is no underlying neurological component. ${ }^{2}$

Appropriate neurological and functional control over the bladder and sphincter coupled with appropriate urogenital development are critical for normal lower urinary tract function. ${ }^{3}$ As most children develop an adult-like pattern of urinary control by ages 4-5 years old, ${ }^{3,4}$ children start being diagnosed with DV around 5 years of age and older. ${ }^{5}$ Patients with DV often have a heterogenous clinical presentation.
Symptoms commonly include lower urinary tract symptoms (frequency, urgency, dysuria), urinary and fecal incontinence, and constipation. ${ }^{1,5}$ According to the International Children's Continence Society (ICCS) guidelines, the gold standard for diagnosis of DV includes repeated uroflow measurements demonstrating a staccato pattern, or urodynamics demonstrating a dysfunctional voiding pattern. ${ }^{6}$ Currently employed first-line treatment strategies of DV include constipation management and urotherapy. ${ }^{7}$ Urotherapy encompasses the education of both the child and caregivers, adequate hydration, timed voiding, proper toilet posturing, and pelvic floor muscle awareness to increase relaxation and contraction of muscles appropriately. ${ }^{8}$

The urge to void can be affected by many factors, both psychological and physiological. One such factor is the presence of bladder irritants. While individuals may have several irritants present in the bladder, the effect seen clinically is often dependent on the concentration of these irritants. Improper hydration affects both the specific gravity of the urine and the concentration of any present urinary irritants, 


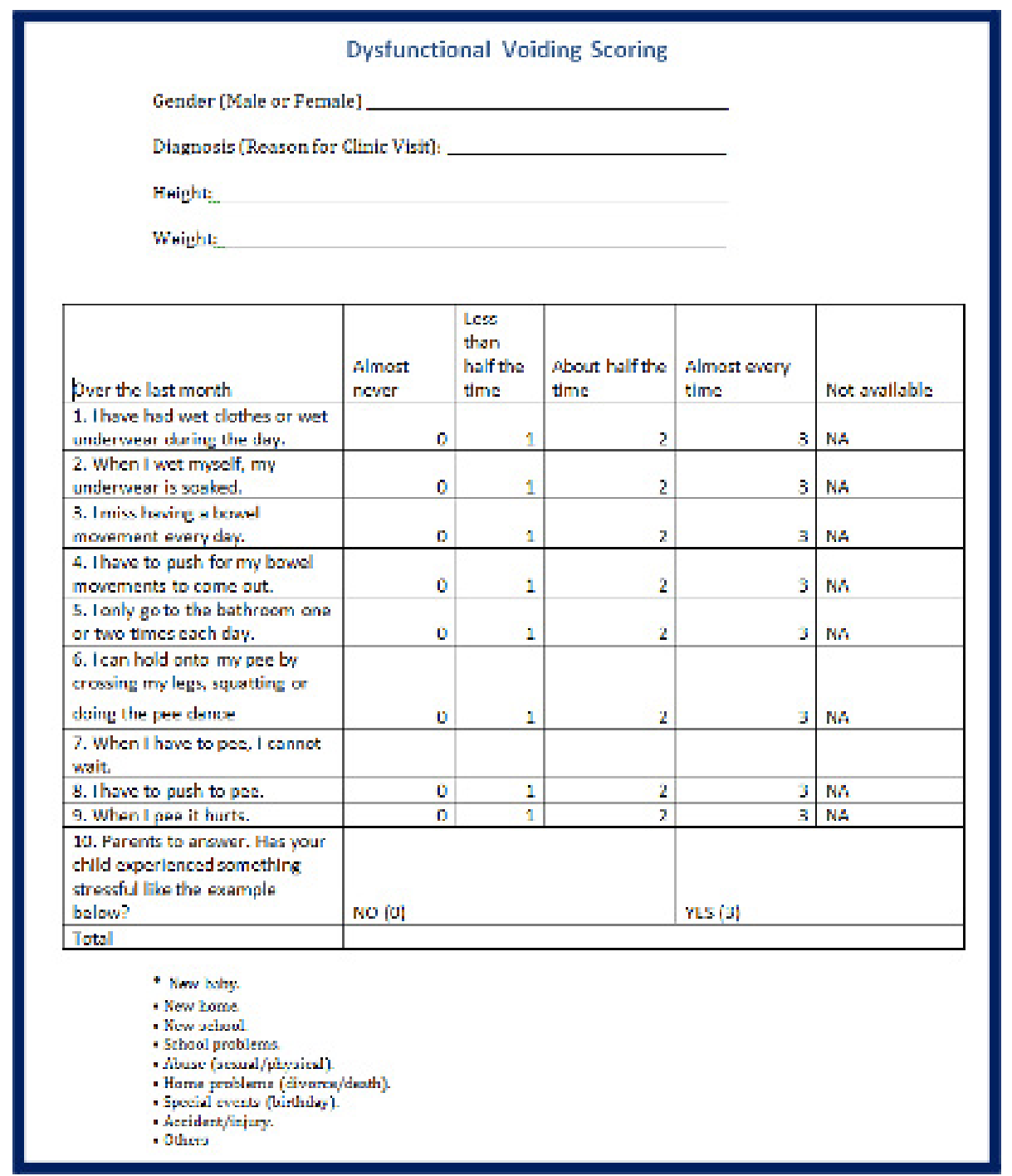

chart to compare hydration status and urinary symptoms between pediatric DV patients and pediatric patients presenting with non-urologic concerns.

\section{METHODS}

\section{Patient Recruitment}

Following Institutional Review Board approval, pediatric patients were prospectively recruited into either the study group or the control group. The study group consisted of pediatric patients $(<18 \mathrm{y} / \mathrm{o})$ who presented to urology clinic for dysfunctional voiding. Patients were excluded from the study group if they had any other urologic diagnosis including an active urinary tract infection, vesicoureteral reflux, or ureteral obstruction. Patients were also excluded if any neurologic diagnosis was present, including spina bifida. The control group consisted of pediatric patients $(<18 \mathrm{y} / \mathrm{o})$ who presented to a

FIGURE 1. The Dysfunctional Voiding Symptom Score (DVSS) Survey.

which can result in many urologic symptoms similar to those experienced in DV patients. ${ }^{9}$ Such symptoms include urinary urgency, frequency, and even incontinence. ${ }^{9}$ Additionally, water intake has been linked to other genitourinary conditions. Adequate daily water intake has been shown to be an effective preventative strategy against recurrent cystitis. ${ }^{10}$

We hypothesize that many DV patients may have some degree of dehydration adding to their urinary symptoms, and that patients with worse dehydration will experience worse urinary symptoms. Herein, we utilized a validated dysfunction voiding questionnaire and a validated dehydration severity primary care physician for a well-check appointment. Patients were excluded if they had any urologic diagnoses or if they had a urologic concern at the well check appointment. All patients included in this study were toilet trained. Informed consent for participation in this study was received from a parent or legal guardian of each patient in both the study and control group.

\section{DAta Retrieval}

Demographic information was obtained from all patients in both the study and control groups, including patient age, sex, height, weight, and BMI. Patients in both groups were administered the Dysfunctional Voiding Symptom Score (DVSS; Figure 1), a validated questionnaire used to evaluate both qualitative and quantitative aspects of urinary 


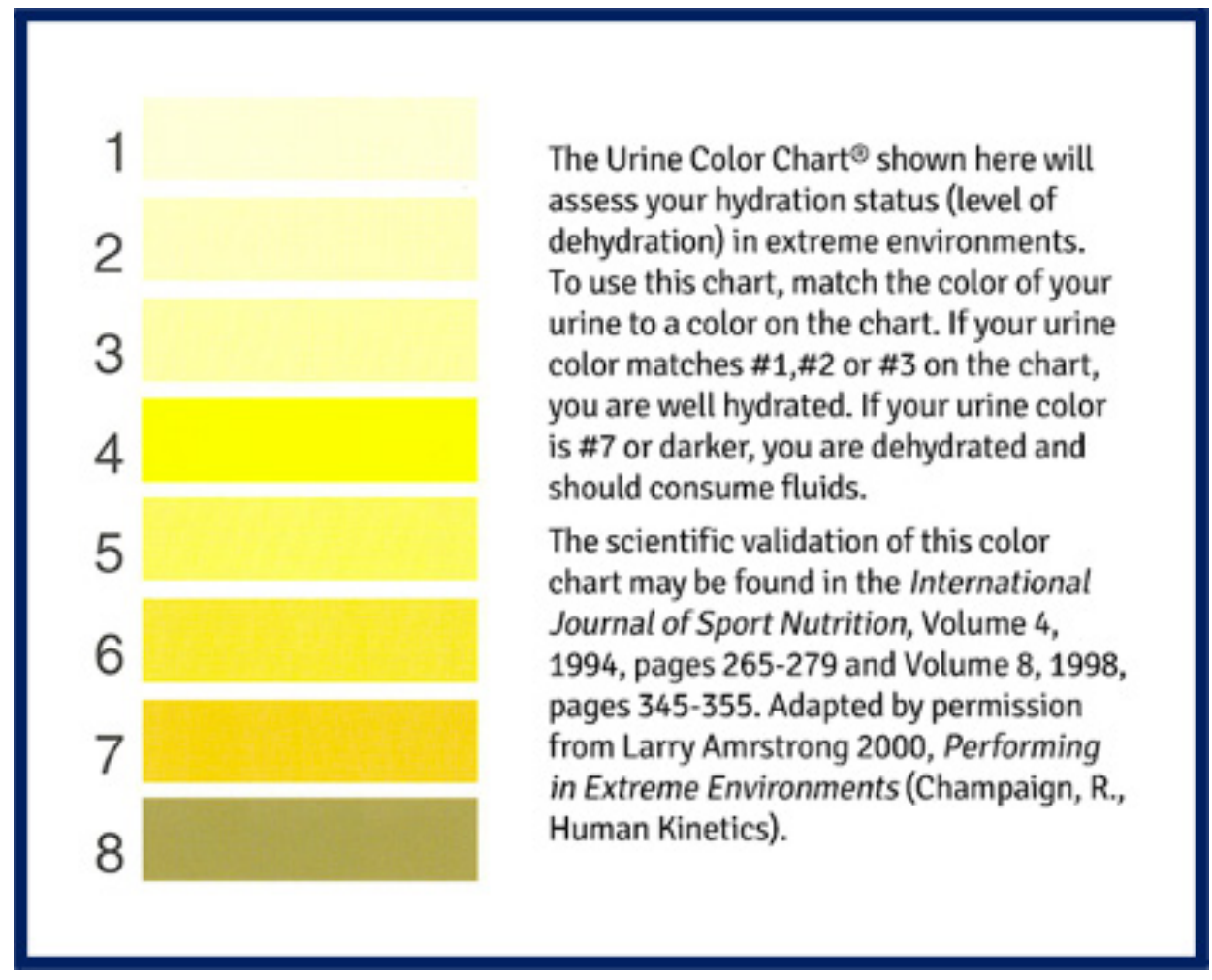

study and control groups. Data was analyzed on an item-for-item basis for each individual question in the DVSS as well as by the total DVSS between both groups. Student 2-tailed t-tests were performed to compare means for continuous variables, and chi-squared tests were used for categorical variable comparison. Statistical significance was defined as $p$ $<0.05$.

FIGURE 2. The Urine Color Chart (UCC).

\begin{tabular}{|c|c|c|c|}
\hline Demographic Variable & $\begin{array}{c}\text { Study Group (DV) } \\
\text { Mean } \pm \text { Std Dev }\end{array}$ & $\begin{array}{c}\text { Control Group } \\
\text { Mean } \pm \text { Std Dev }\end{array}$ & P Value \\
\hline Age & $9.724 \pm 3.731$ & $9.0952 \pm 4.158$ & 0.587 \\
\hline Gender & $0.310 \pm 0.471$ & $0.524 \pm 0.512$ & 0.140 \\
\hline Height & $54.931 \pm 9.217$ & $53.534 \pm 8.853$ & 0.594 \\
\hline Weight & $102.517 \pm 54.565$ & $91.915 \pm 38.935$ & 0.431 \\
\hline BMI & $22.1 \pm 6.645$ & $21.2 \pm 3.972$ & 0.551 \\
\hline
\end{tabular}

TABLE 1. Mean values for demographic variables in the study group and control group.

\section{RESULTS}

symptoms. Patients in both groups were then administered the Urine Color Chart (UCC; Figure 2), a validated scale to quantify hydration status based on the color of the patient's urine.

\section{Statistical Data Analysis}

Mean demographic data (age, gender, height, weight, and $\mathrm{BMI}$ ) were compared between the treatment and study groups. Mean urologic data obtained from the DVSS and hydration status data from the UCC were then compared between the

\section{Demographic Data}

Fifty total pediatric patients were recruited into the study from 2016-2018. Twenty-nine DV patients were enrolled into the study group and 21 nonurologic pediatric patients were enrolled into the control group. Both patient groups were equivalent with regards to age, gender, height, weight, and BMI (Table $1 ; p>0.05$ ), indicating successful randomization of the study and control groups. Children in the dysfunction voiding group ranged in age from 3-15 with an average age of 9.72. Nine patients were male and 20 were female. All 29 


\begin{tabular}{|c|c|c|c|}
\hline DVSS Item & $\begin{array}{c}\text { Study Group (DV) } \\
\text { Mean } \pm \text { Std Dev }\end{array}$ & $\begin{array}{c}\text { Control Group } \\
\text { Mean } \pm \text { Std Dev }\end{array}$ & P Value \\
\hline Item 1 & $0.483 \pm 0.969$ & $0.0952 \pm 0.301$ & 0.0543 \\
\hline Item 2 & $1.345 \pm 1.396$ & $0.0952 \pm 0.301$ & 0.0000667 \\
\hline Item 3 & $0.862 \pm 1.0413$ & $0.381 \pm 0.590$ & 0.0465 \\
\hline Item 4 & $1.345 \pm 1.1530$ & $0.667 \pm 0.796$ & 0.0187 \\
\hline Item 5 & $0.759 \pm 1.1643$ & $0.476 \pm 0.873$ & 0.337 \\
\hline Item 6 & $1.586 \pm 1.1602$ & $2.286 \pm 1.231$ & 0.0500 \\
\hline Item 7 & $1.276 \pm 1.257$ & $0.571 \pm 0.870$ & 0.0248 \\
\hline Item 8 & $0.448 \pm 0.770$ & $0.0952 \pm 0.436$ & 0.0481 \\
\hline Item 9 & $0.931 \pm 0.980$ & $0.190 \pm 0.512$ & 0.00135 \\
\hline Item 10 & $0.931 \pm 1.388$ & $1.286 \pm 1.521$ & 0.407 \\
\hline Total Score & $9.966 \pm 4.522$ & $6.143 \pm 2.330$ & 0.000388 \\
\hline
\end{tabular}

TABLE 2. Mean values for DVSS individual items and total DVSS score in the study group and control group.

\section{DVSS Respose To Questions}

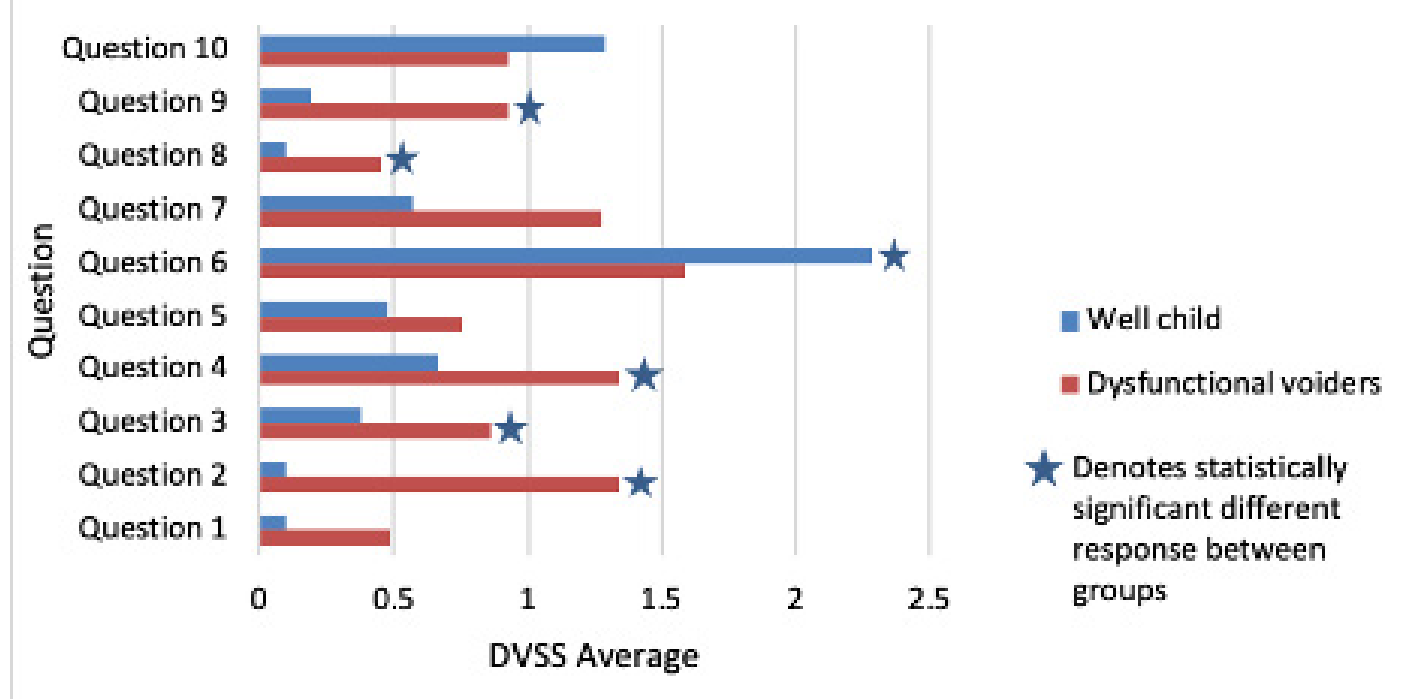

FIGURE 3. DVSS Responses by Question. This bar graph compares the average numerical value of the response by patients in the dysfunctional voiding group (red) and the well child group (blue) for each question of the DVSS.

patients included in the DV group were Caucasian. In the non-urologic pediatric patient group, children ranged in age from 2-17 with an average age of 9.09. There were 11 males and 10 females in this group. The ethnicity/races of participants in the non-DV group included 1 Hispanic, 1 African American, and 20 Caucasian patients.

\section{UROLOGIC DATA}

The study group (DV patients) had significantly higher scores in 7 out of 10 individual items within the DVSS when compared to the control group
(Table 2; Figure 3; $p<0.05$ ). The study group patients additionally had significantly higher total scores on the DVSS than non-urologic patients (Table 2; Figure $3 ; p=0.0004)$.

\section{HydRATION StATUS DATA}

There was no significant difference between UCC scores between the study group and the control group (Figure $4 ; p=0.753$ ). A linear regression analysis was performed and demonstrated a linear relationship between DVSS and UCC scores (Figure 5). 


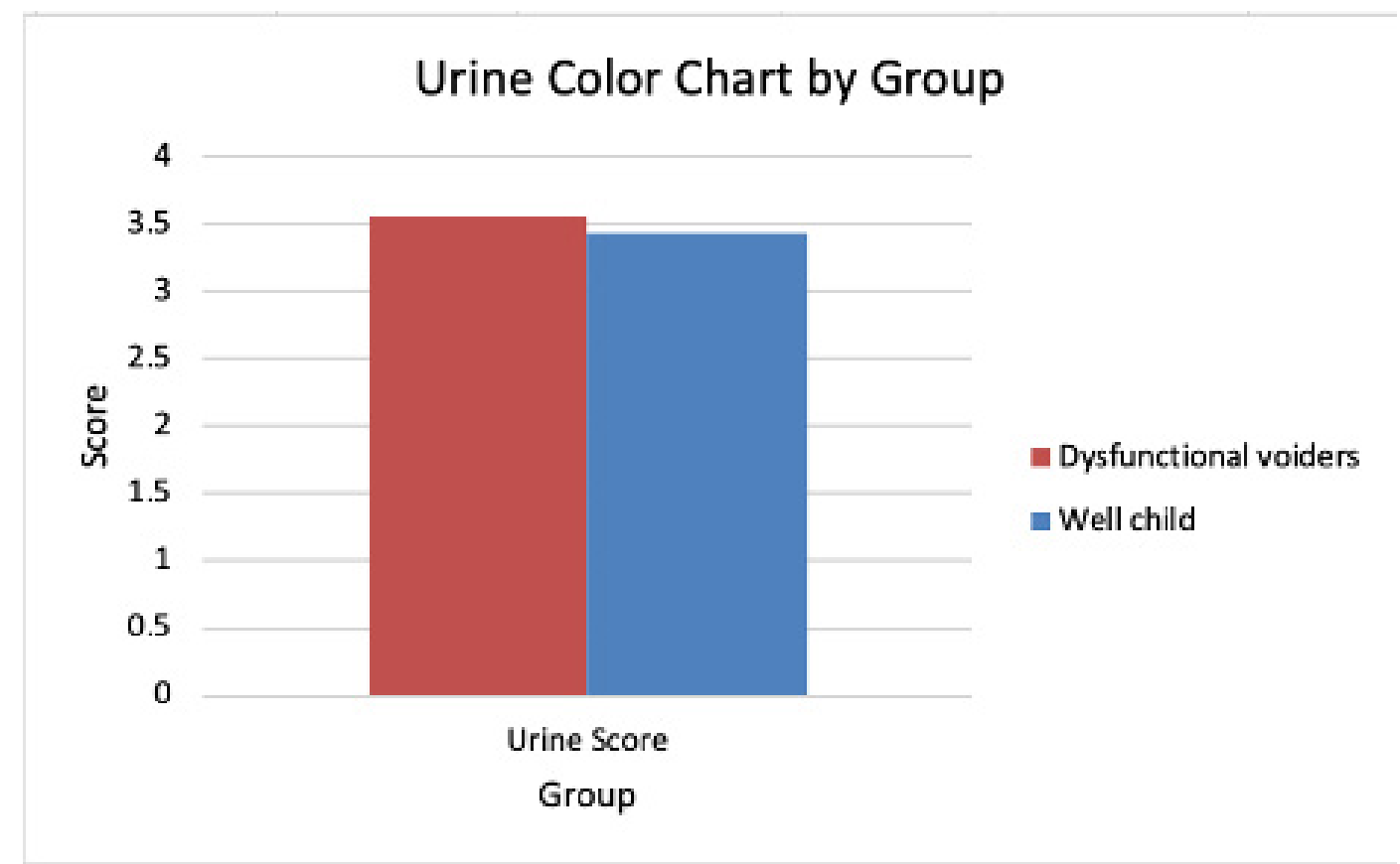

FIGURE 4. Urine Color Chart by Group. This bar graph compares the average reported numerical value of the urine color (based on the numerical value associated with urine color from the Urine Color Chart) between the dysfunctional voiding group (red) and the well child group (blue).

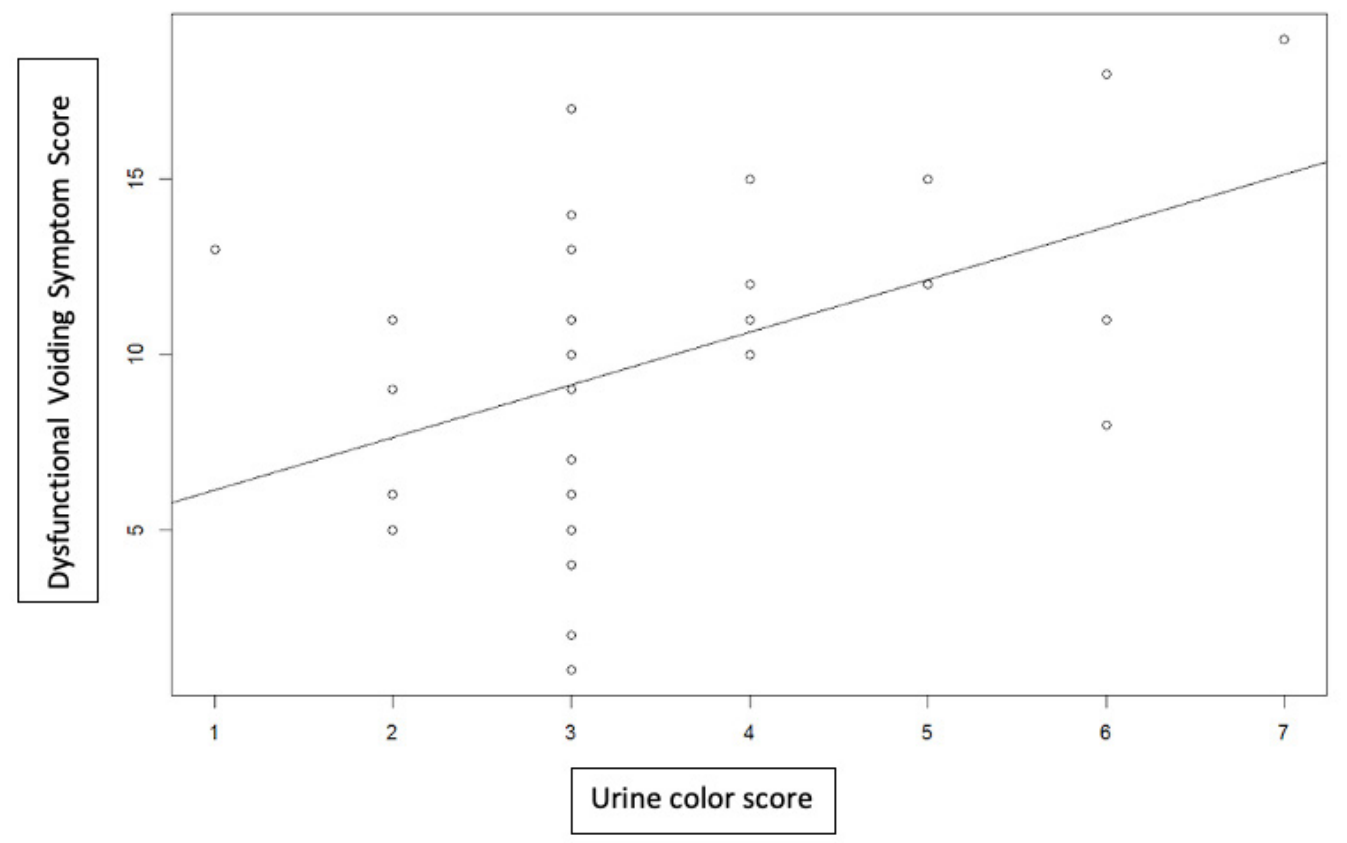

FIGURE 5. This graph depicts the dysfunction voiding symptom survey scores vs the urine color scores for the individuals in the dysfunctional voiding cohort. 


\section{DISCUSSION}

To date, diagnosis and management of pediatric DV patients has been mostly reliant on clinical diagnoses and symptom control due to the invasiveness of diagnostic procedures such as urodynamics. ${ }^{7}$ The utilization of non-invasive tools such as the DVSS to gauge impact of attempted therapeutic strategies has helped to objectively monitor for improvement and symptom resolution in affected patients. In this present study, study group (DV) patients demonstrated higher DVSS scores, indicating significantly worse DV/urologic symptoms as compared to non-urologic control patients. Additionally, study group (DV) patients demonstrated higher DVSS individual item scores for 7 out of the 10 DVSS items: specifically, items $2,3,4,6,7,8$, and 9 (Table 1). DVSS items that were significantly higher in the study group patients included questions about urine and fecal incontinence, constipation, and dysuria (Figure 1). DVSS item 10 asks the parents to indicate whether the child has experienced a stressful life circumstance (Figure 1). DVSS item 10 scores were not significantly different between the study and control groups, indicating the patient populations were matched with regard to previous life stressors.

While there was no significant difference in mean UCC scores between the study and control groups, a regression analysis indicated a linear relationship between UCC and DVSS scores such that UCC indicating worse hydration status correlated positively with DVSS scores indicating worse DV symptoms. A limitation of this study is the use of only one validated hydration scoring system for indicating hydration status. The UCC requires the patient and/ or guardian to indicate the most common color of urination the child experiences. Further studies should implement multiple modalities of hydration status quantification. For example, specific gravity of urine is a non-invasive and inexpensive tool for quantifying urine concentration. Another limitation of the study is that it was performed at a single institute with a smaller sample size. Further studies should be performed across multiple institutions with a larger number of patients recruited.

This study demonstrates that DV patients often suffer from more than just one symptom. This study also demonstrates a linear relationship between hydration status and DVSS scores, indicating the clinical necessity to educate both the patients and their families on the importance of adequate daily hydration. An additional element that could be addressed in further studies is comparing patients with dysfunctional voiding before and after intervention to children without urologic complaints overtime. Sometimes care providers are more apt to notice symptoms once they are thinking about them, therefore it would be interesting to see if the symptoms experienced by the well child pediatric patients would be reported differently overtime while also monitoring how symptom scores change for dysfunctional voiding patients undergoing treatment.

\section{SUPPLEMENTARY DESCRIPTION}

The supplementary data file includes a table demonstrating the distribution of demographic variables in the study group and control group, a table with the mean values for DVSS individual items and total DVSS score in the study group and control group, the Dysfunctional Voiding Symptom Score (DVSS) Survey, and the Urine Color Chart (UCC).

KEY MESSAGES

1. DV population had significantly higher scores in 7 out of 10 individual items within the DVSS when compared to those presenting for nonurologic complaints $(p<0.05)$. Our data backs the DVSS as a valid tool for distinction between normal voiders and dysfunctional voiders.

2. No significant difference was noted in UCC scores between patient groups $(p=0.753)$. Therefore, our data shows this as less predictive of dysfunctional voiding vs normal voiding.

3. The DV group patients had significantly higher total scores on the DVSS than non-urologic patients (Table 2; Figure 3; $p=0.0004$ ), further validating the DVSS as a valid tool for distinction between normal voiders and dysfunctional voiders.

4. Within the dysfunctional voiding group, there was a linear relationship between DVSS and UCC scores. Since some symptoms of DV can be additionally associated with dehydration, 
additional research could be performed to further tease out the relationship between these variables such as with a blind double cross over study.

5. Both patient groups evaluated in this study (DV vs non DV) were equivalent with regards to age, gender, height, weight, and BMI (Table 1; p > $0.05)$, indicating successful randomization of the study and control groups.

\section{AUTHOR AFFILIATIONS}

1. West Virginia University, Department of Urology, Morgantown, West Virginia

2. West Virginia University, Department of Pediatrics, Morgantown, West Virginia

\section{REFERENCES}

1. Sinha S. Dysfunctional voiding: a review of the terminology, presentation, evaluation and management in children and adults. Indian Journal of Urology 2011; 27(4):437-447.

2. Leclair MD, Heloury Y. Non-neurogenic elimination disorders in children. Journal of pediatric urology 2010; 6(4):338-345.

3. Fuentes $M$, Magalhaes J, Barroso U. Diagnosis and management of bladder dysfunction in neurologically normal children. Frontiers in pediatrics 2019; 7:298.

4. Skobejko-Wlodarska L. Non-neurogenic lower urinary tract dysfunction. Pol Merkur Lekarski 2008; 24:131-7.

5. O'Brien M. Dysfunctional Voiding and Other Daytime Wetting Disorders. Pediatric Urology Book [Internet]. Available from: http://www. pediatricurologybook.com/dysfunc void.html

6. Chase J, Austin P, Hoebeke P, McKenna P. The management of dysfunctional voiding in children: from the standardization committee of the International Children's Continence Society. Journal of Urology 2009; 183:1296-1302.

7. Santos JD, Lopes RI, Koyle MA. Bladder and bowel dysfunction in children: an update on the diagnosis and treatment of a common, but underdiagnosed pediatric problem. Canadian Urological Association 2017; 11:72.

8. Hagstroem S, Rittig S, Kamperis K, Djurhuus JC.
Timer watch assisted urotherapy in children: a randomized controlled trial. Journal of Urology 2010; 184(4):1482-8

9. Hooton TM, Vecchio M, Iroz A, et al. Effect of increased daily water intake in premenopausal women with recurrent urinary tract infections: a randomized clinical trial. JAMA Internal Medicine 2018; 178(11): 1509-1515.

10. Farhat W, Bagli DJ, Capolicchio G, et al. Bladder function - dysfunctional voiding scoring system: quantitative standardization of dysfunctional voiding symptoms in children. Journal of Urology 2000; 164(3):1011. 